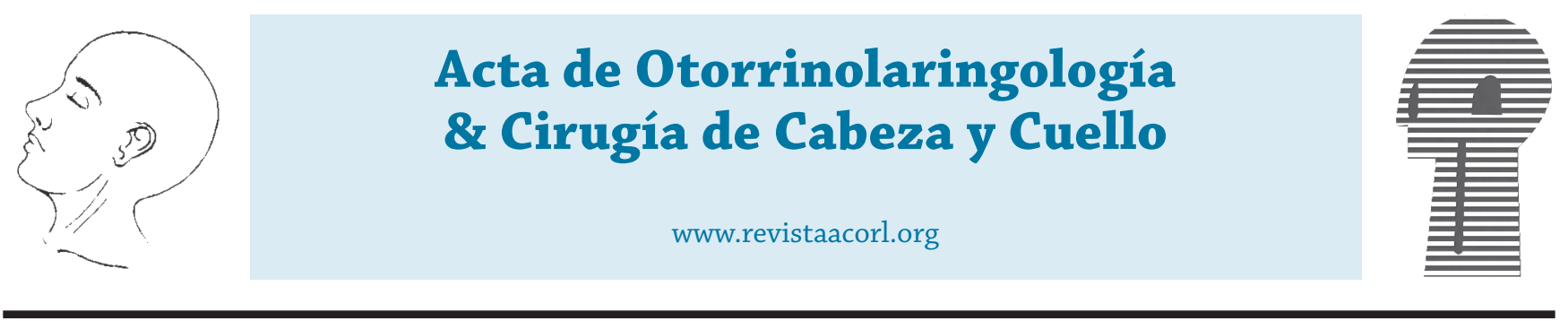

\title{
Síndrome de Lemierre simulando síndrome paraneoplásico: reporte de un caso y revisión de la literatura
}

\section{Lemierre syndrome simulating signs and symptoms of paraneoplastic syndrome: case report and literature review.}

Katherine Re dondo De Oro*, César Redondo Bermúdez*, Luis DavidMendoza Durán**, John Maldonado Brigante**.

* Médico Patólogo. Docente de la Facultad de Medicina. Grupo de investigación en Histopatología, Universidad de Cartagena.

** Estudiante de Pregrado, Facultad de Medicina, Universidad de Cartagena.

Forma de citar: Redondo-de Oro KT, Redondo-Bermúdez C, Mendoza-Durán LD, Maldonado-Brigante JM. Síndrome de Lemierre simulando síndrome paraneoplásico: reporte de un caso y revisión de la literatura. Acta otorrinolaringol. cir. cabeza cuello. 2018;47(3):178-182.Doi: 10.37076/acorl.v47i3.444

\section{INFORMACIÓN DEL ARTÍCULO}

Historia del artículo:

Recibido: 2 de Agosto de 2019

Evaluado: 16 Septiembre de 2019

Aceptado: 30 Septiembre de 2019

Palabras clave (DeCS):

Síndrome de Lemierre;

Tromboflebitis; Fusobacterium.

\section{RESUMEN}

El síndrome de Lemierre es una enfermedad rara, ocasionada por la complicación grave de una infección orofaringea que se manifiesta con una tromboflebitis séptica en la yugular interna y una embolización séptica a distancia. En principio, el síndrome cursa con fiebre, dolor en el área del ángulo de la mandíbula, inflamación de la región periamigdalina y un crecimiento unilateral del cuello luego de una infección en las estructuras de la cabeza y el cuello. En el artículo se presenta el caso de una mujer de 45 años quien consultó por una complicación infecciosa posterior a la realización de una exodoncia. El diagnóstico clínico se realizó con base en los signos y síntomas que indicaban tromboflebitis séptica del seno cavernoso. Se realiza antibióticoterapia con resultados positivos en la paciente $y$, como resultado, una mejoría total del estado de salud. Se presenta este caso por lo infrecuente de la entidad.

\section{Correspondencia:}

Luis David Mendoza Durán

Correo electrónico: luisdmendozadu@gmail.com

Facultad de Medicina, Universidad de Cartagena. Carrera 50a \# 24- 63, Provincia de Cartagena, Bolívar. 


\section{ABSTRACT}

Key words (MeSH):

Lemierre Syndrome;

Thrombophlebitis; Fusobacterium.
Lemierre syndrome is a disease caused by the serious complication of an oropharyngeal infection that manifests with septic thrombophlebitis in internal jugular and remote septic embolization. It presents with fever, pain in the area of the jaw angle, inflammation of the peritonsillar region and unilateral neck growth after an infection in the head and neck structures. We present the case of a 45-year-old woman who consulted due to an infectious complication after carrying out the extraction. The clinical diagnosis was made based on the signs and symptoms that indicated septic thrombophlebitis of the cavernous sinus. Antibiotic therapy is performed with positive results in the patient and total improvement of the state of health.

\section{Introducción}

El síndrome de Lemierre, también conocido como tromboflebitis séptica de la vena yugular interna, septicemia posanginal o necrobacilosis (1), es una patología poco frecuente que afecta a una por cada millón de personas al año, con mayor frecuencia a adolescentes (entre 16 y 19 años) y adultos (2). Fue descrita por primera vez por André Lemierre en 1936, y se caracteriza por una septicemia secundaria a una infección de alguna de las estructuras de la cabeza y el cuello, particularmente de la orofaringe, que se disemina complicándose hacia una tromboflebitis supurativa ipsilateral de la vena yugular interna, la cual tiende a propagarse mediante émbolos hacia otras partes del organismo. El síndrome de Lemierre se manifiesta con fiebre elevada acompañada de temblores y sudoración profusa, dolor en el área del ángulo de la mandíbula, disfagia, disfonía, inflamación de la región periamigdalina, rigidez y crecimiento unilateral del cuello. La infección se relaciona con la bacteria gramnegativa Fusobacterium necrophorum en el $81,7 \%$ de los casos.

\section{Caso clínico}

Paciente femenina de 45 años, quien consulta por un cuadro clínico posterior a una exodoncia en los órganos dentarios 24 y 25 con sangrado gingival, edema, dolor en piso de la lengua, fiebre, escalofríos y secreción purulenta por orificio de extracción, que empeoró con el tiempo y dificultó la masticación, y está asociada con una hipoacusia bilateral y una secreción purulenta bilateral por los conductos auditivos. Al examen físico impresionó absceso por gérmenes comunes, por lo que se manejó con antibióticos orales y analgésicos de forma ambulatoria. Posterior a esto, aparece una lesión tumoral en la hemicara derecha, cierre palpebral y lagrimeo bilateral ocular seguido de una celulitis periorbitaria, por lo que se decide dar manejo intrahospitalario con penicilina $\mathrm{y}$ clindamicina.

Durante su evolución, la paciente presenta picos febriles, por lo que se decide realizar hemocultivo y se escalona antibioticoterapia con piperacilina, tazobactam y vancomicina. Ante los hallazgos descritos se considera la realización de una biopsia de la lesión en la encía vestibular a nivel de los órganos dentarios 24 y 25, y un drenaje del absceso localizado a nivel de la mucosa labial inferior. En días siguientes aparecen episodios de dificultad respiratoria, por lo que se opta por realizar una radiografía de tórax que demuestra radiopacidad apical derecha, lo cual da como impresión clínica una neoplasia maligna (Figura 1).

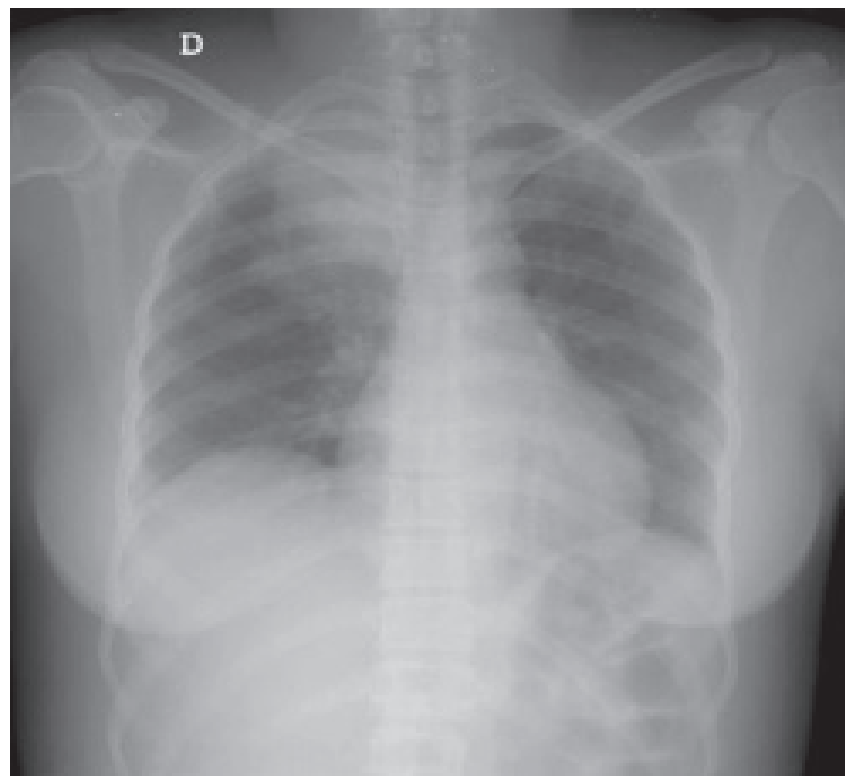

Figura 1. Rx de tórax PA donde se evidencia imagen radiodensa redondeada apical derecha, de márgenes bien definidos de 53×55 $\mathrm{mm}$ de diámetro.

En el reporte anatomopatológico de la biopsia incisional de la lesión en la encía palatina y vestibular se encontró una lesión vestibular ampollosa subepitelial, compatible con penfigoide, por lo que se sospecha que el cuadro era secundario a una lesión neoplásica a nivel pulmonar; luego de esto, se ordenan marcadores tumorales que se reportan como negativos. Se realiza una tomografía axial computarizada (TAC) de tórax simple y contrastado que reporta una formación sólida con aspecto de masa de bordes irregulares (43 x $63 \mathrm{~mm}) \mathrm{de}$ diámetros mayores con áreas de necrosis en su interior. Esto indica un probable origen neoplásico (Figura 2). 

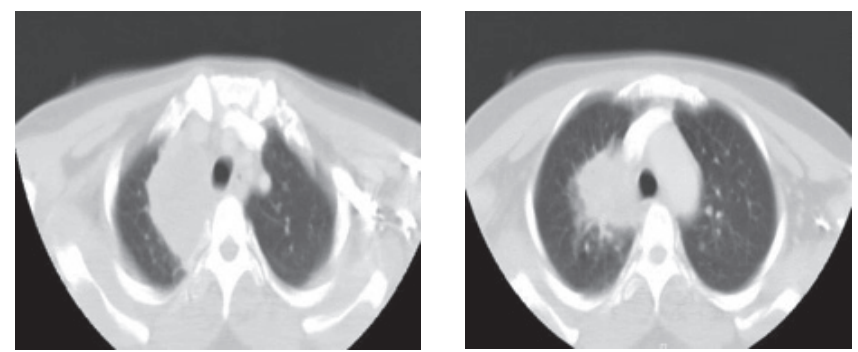

Figura 2. Tomografía de tórax con donde se evidencia formación sólida con aspecto de masa de bordes irregulares de $43 \times 66 \mathrm{~mm}$ de diámetro.

Ante esta sospecha, se decide realizar una resección de la lesión pulmonar por videotoracoscopia en cuña del ápice pulmonar derecho y es enviado al servicio de patología. En el reporte de esta muestra se observa un parénquima pulmonar comprometido difusamente por un proceso necrotizante extenso e inflamación crónica mixta; en la valoración morfológica no se observa malignidad ni microorganismos (Figura 3).
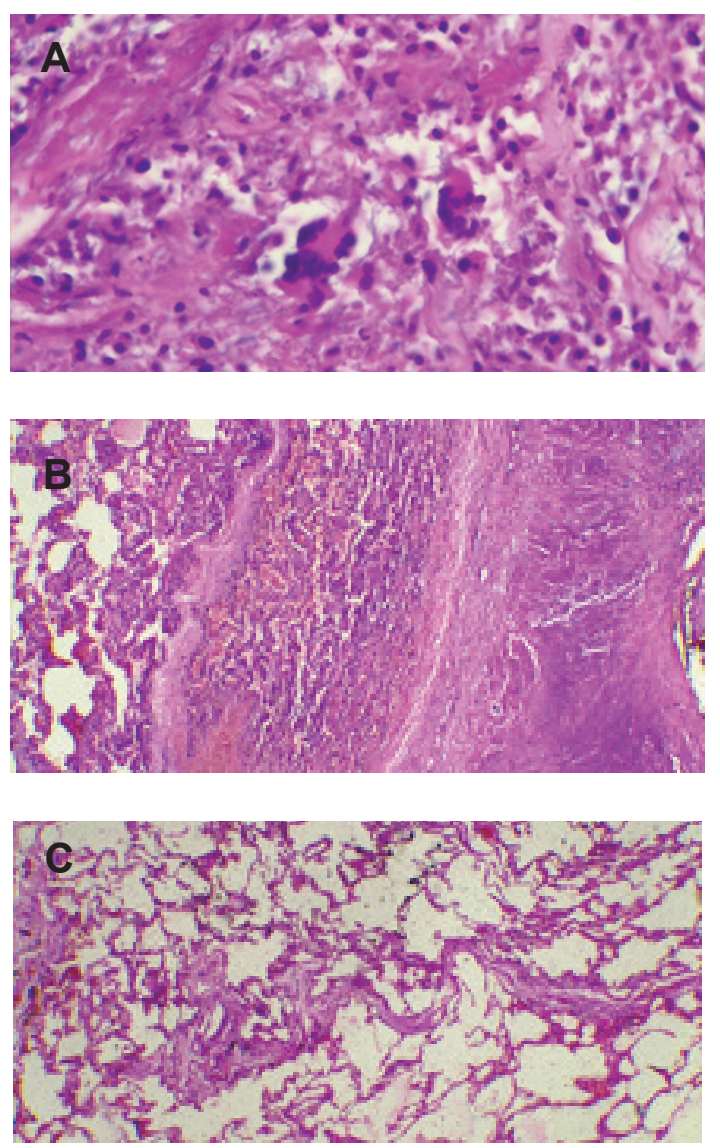

Figura 3. Parénquima pulmonar comprometido por un proceso inflamatorio crónico con formación de granulomas y extensas áreas de necrosis. Se evidencian las células gigantes multinucleadas de tipo cuerpo extraño. No se reconocen microorganismos. (A) H-E 40X. (B) H-E 10X (C) Parénquima pulmonar vecino sano.
Los estudios de inmunohistoquímica confirmaron la naturaleza reactiva de la lesión, y los estudios de histoquímica para hongos y micobacterias fue negativo (PAS, ZN y GOMORI). La relación de los hallazgos del departamento de patología, sumada a las manifestaciones clínicas presentadas por la paciente, obliga a considerar el síndrome de Lemierre. No se logró cultivar el microorganismo responsable de la mayoría de los casos, pero el descartar otro tipo de gérmenes y la presentación clínica de la enfermedad obligan a considerarlo como agente etiopatogénico de este caso.

\section{Discusión}

El síndrome de Lemierre es una rara enfermedad que se caracteriza por la inflamación agresiva del espacio parafaríngeo lateral, tromboflebitis de la vena yugular interna y el desarrollo de los émbolos sépticos a distancia (3). Este cuadro suele presentarse en adultos jóvenes sanos, sin déficit inmunitario, hábitos tóxicos u otros factores de riesgo predisponentes. Sus complicaciones pueden conllevar una considerable tasa de mortalidad, especialmente cuando el tratamiento con antibióticos se retrasa. Otras complicaciones menos graves incluyen infecciones metastásicas en articulaciones, órganos internos y/o el cerebro (4).

Etiológicamente, Fusobacterium necrophorum se aísla como agente responsable en el $81,7 \%$ de los casos, un germen habitual de la flora oral, intestinal y genital. En general, se trata de un anaerobio estricto y gramnegativo. Paralelamente, existen otros organismos implicados que pueden ser aislados $(5 \%)$ o presentarse en combinación con $F$. necrophorum $(10$ $\%$ ), como diferentes tipos de Bacteroides (asaccharolyticus, fragilis), Peptostreptococcus, Streptococcus del grupo B y C, Staphylococcus epidermidis, Enterococcus spp., Proteus mirabilis, Eubacterium spp., Eikenella corrodens, y Candida spp., e incluso Staphylococcus aureus. Se han descrito también factores predisponentes para la infección por fusobacterias consistentes con alteraciones de las barreras de defensa cutáneo-mucosas e incluso faringitis producidas por el virus de Epstein-Barr $(3,5)$.

En la mayoría de los casos, se trata de jóvenes con infección faríngea que no remite pasados 3-7 días, y se complica con la aparición de fiebre alta, sepsis y la aparición de signos de flogosis del espacio parafaríngeo lateral con tromboflebitis de la vena yugular interna y, finalmente, focos sépticos a distancia (6). La patogénesis y clínica de esta enfermedad progresa en varias etapas. La primera es la infección primaria, que la mayoría de las veces consiste en una faringitis aguda. Las amígdalas palatinas y el tejido periamigdalino son el sitio predominante de infección primaria $(87,1 \%)$; se han reportado casos secundarios a infecciones dentarias, mastoiditis, parotiditis, sinusitis, otitis y de piel y tejido celular subcutáneo de cabeza y cuello. La clínica en este estadio depende del sitio de inicio de la infección $(3,5)$.

La segunda etapa consiste en la invasión del espacio faríngeo, principalmente por vía linfática pero también por extensión perivascular. Es en este estadio donde puede com- 
plicarse con tromboflebitis de la vena yugular interna. Las manifestaciones clínicas van a variar según la invasión y el compromiso de las estructuras musculares, vasculares y nerviosas allí alojadas. El signo más frecuente y alarmante de este cuadro en pacientes con faringitis aguda es la inflamación dolorosa del cuello, induración del ángulo mandibular y músculo esternocleidomastoideo, que corresponde a la tromboflebitis de la yugular interna (3).

El tercer estadio es la infección metastásica, en la que se produce bacteriemia y extensión hematógena. El sitio más común de embolización séptica es el pulmón (80-90 \% de los casos), lo que se traduce radiológicamente como infiltrados bibasales, derrames pleurales, cavitaciones, nódulos difusos, empiemas, abscesos pulmonares y neumotórax. En segundo lugar, se encuentran las articulaciones (15-20\%), siendo más afectadas las caderas, los hombros y las rodillas en forma de artritis. Otros sitios de diseminación séptica son el músculo, el sistema nervioso central (SNC) y el tracto gastrointestinal $(3,5)$.

La ausencia de trombosis yugular interna no excluye el diagnóstico de Lemierre, pues esta puede ocurrir en gran variedad de venas del cráneo y/o el cuello; se han descrito casos que presentan trombosis del seno cavernoso $(7,8)$.

El diagnóstico es básicamente clínico y puede ser difícil por la rareza del cuadro, aunque debe sospecharse ante toda sepsis grave con antecedente de infección orofaríngea reciente (9). El apoyo en la imagenología como la ecografía Doppler color y el TAC cervical con medio de contraste son útiles para documentar las anomalías anatómicas subyacentes, como edema o absceso del espacio parafaríngeo lateral $\mathrm{y}$ trombosis de la vena yugular interna, pero se considera el TAC cervical como la prueba de imagen de elección para detectar dichas anomalías (6).

En la evaluación de embolias sépticas es importante mencionar que los hallazgos radiológicos, particularmente en la TAC de tórax, pueden estar presentes incluso antes que los hemocultivos estén positivos. Ante la sospecha de trombosis de la vena yugular interna, la ecografía Doppler cervical es el estudio de primera línea con una sensibilidad y especificidad de $92 \%$ y $98 \%$, respectivamente. La ausencia de flujo al Doppler color y la falta de compresibilidad de la vena representan hallazgos de alta sensibilidad, mientras que el signo más específico es la visualización del trombo $(10,11)$.

El tratamiento se basa en la administración de antibióticos por vía venosa, seguidos por un período de terapia oral prolongada. El Fusobacterium es clásicamente sensible a la penicilina $\mathrm{G}$ y otros antibióticos con actividad anaerobicida, como el metronidazol, la clindamicina y el imipenem. La susceptibilidad a las cefalosporinas, la eritromicina y las tetraciclinas es variable, y es resistente al aztreonam y el trimetopin-sulfametoxazol, como también a aminoglucósidos (6). En 1990, Appelbaum y colaboradores encontraron que el $40 \%$ de las Fusobacterium aisladas producían $\beta$-lactamasas, por lo que se recomienda el uso de antibióticos resistentes a las $\beta$-lactamasas con actividad anaeróbica (12).
La terapia quirúrgica puede ser necesaria en ciertas situaciones. Si hay descompensación del paciente o falta de mejora, se debe considerar la terapia quirúrgica. Esto incluye pacientes con sepsis incontrolada severa o insuficiencia pulmonar a pesar del tratamiento médico óptimo. Esto podría incluir drenaje de un absceso, ligadura venosa o resección venosa (13).

Es controvertido el uso de terapia anticoagulante. Algunos autores argumentan que con dicha terapia existe el riesgo, al menos teórico, de facilitar la diseminación de la infección. Por otro lado, hay quien defiende su uso basado en los buenos resultados obtenidos en pacientes con tromboflebitis pélvica séptica (5).

\section{Conclusión}

Con la presentación de este caso, se evidencia un caso inusual de síndrome de Lemierre en el seno cavernoso secundario a una infección dentaria. La localización, microorganismo e infecciones causales del síndrome de Lemierre pueden variar en ocasiones, lo que amerita el diagnóstico inmediato a partir de los signos y síntomas, acompañados de estudios imagenológicos y cultivos para la identificación del microorganismo, y así tratar oportunamente al paciente con antibióticos específicos y, de ser necesario, intervención quirúrgica.

\section{Conflictos de intereses}

Los autores declaran no tener conflictos de intereses.

\section{REFERENCIAS}

1. Dibildox Martínez J. [Insertar título del capítulo]. En: Temas selectos en otorrinolaringología y cirugía de cabeza y cuello. México: Prometeo Editores; 2013.

2. O’Dwyer DN, Ryan S, O'Keefe T, Lyons J, Lavelle L, McKone E. Lemierre's syndrome. Ir J Med Sci. 2011;180(2):565-7. doi: 10.1007/s11845-008-0255-7.

3. Tellería Martína A, Jiménez Urraa I, Susperregui Insaustia I, Ramos Castroa J, Gutiérrez Olivera A. Síndrome de Lemierre. Med Intensiva 2005;29(8):441-4.

4. Johannesen KM, Bodtger U. Lemierre's syndrome: current perspectives on diagnosis and management. Infect Drug Resist. 2016 Sep 14;9:221-227.

5. Muñoz Grajales C, Hernández López DL, Gómez Roldán CI. Síndrome de Lemierre: reporte de una variante complicada con trombosis de seno cavernoso. Medicina UPB. 2010;29(1):71-77.

6. Andrade JT, San Martín JT, Grau C. Síndrome de Lemierre, secundario a otitis media aguda: Revisión a partir de un caso. Rev. Otorrinolaringol. Cir. Cabeza Cuello. 2013;73(3):263-267. doi: 10.4067/S0718-48162013000300009.

7. Rudski Ricondo L, Ramos Gómez MJ, Cantador JA, BravoRodríguezFA. Síndrome de Lemierre: trombosis de seno cavernoso y oclusión de arteria carótida interna secundarios a sinusitis esfenoidal aguda. Acta Otorrinolaringol Esp. 2013;64(1):75-77.

8. Samudio Domínguez GC, Irala E, Ruiz-Díaz J. Síndrome de Lemiere por SAMS. Reporte de caso pediátrico. Pediatr. (Asunción). 2015;42(3):225-228. 
9. Carrillo López A, Herrero Cereceda JE, Palmer Sans M, Madrid Vergara ME, Royo Villa C, Molina Povedano MA. Síndrome de Lemierre en paciente adulto con circulación de Fontan. Rev Esp Cardiol. 2017;70:777-9. doi: 10.1016/j.recesp.2016.09.050.

10. Maldonado Sch I, Gutiérrez JM, Wilkens A, Weitz C, Rojas A, Varela C. Síndrome de Lemierre: Una entidad clínica casi en el olvido. Reporte de tres casos y revisión de la literatura. Revista Chilena de Radiología. 2015;21(1):34-40.

11. Golpe R, Marín B, Alonso M. Lemierre's syndrome (necrobacillosis). Postgrad Med J. 1999;75:141-144. doi: 10.1136/ pgmj.75.881.141

12. Winter M, Saavedra A, Grau C, Caro J. Síndrome de Lemierre: reporte de un caso. Rev. Otorrinolaringol. Cir. Cabeza Cuello. 2013;73(2):164-168. doi: 10.4067/S0718-48162013000200009.

13. Charles K, Flinn WR, Neschis DG. Lemierre's syndrome: a potentially fatal complication that may require vascular surgical intervention. J Vasc Surg. 2005;42(5):1023-5. 